\title{
Influence of feedback type on comprehension with two variants of a text
}

\author{
PHILIP LANGER, VERNE KEENAN, and SUSAN NELSON \\ University of Colorado, Boulder, Colorado
}

\begin{abstract}
This study is another in a continuing series on the effects of feedback on text comprehension. Subjects reconstructed scrambled versions of qualitatively similar texts. One version (route) was based on the sequential order of locations encountered in a drive through a mythical town; the other described the same town in geographical terms (survey). The reconstruction process was assisted by information regarding the appropriateness of sentence placement. Feedback was provided in the form of either tokens or access to a map of the town. Results were in line with previous research: (1) Recall of text was superior for the route version and particularly with map assistance, (2) discrimination between paraphrases and original sentences in the passage was not a function of either version or feedback, and (3) concordance between the reconstructed passage sentence order and the original passage was not related to either recall or recognition. The results support previous assumptions regarding the specificity of effects for text content and feedback.
\end{abstract}

Within the field of instructional psychology, the belief that assistance in the learning process is better than no assistance approaches the level of an axiomatic truth (Berliner \& Rosenshine, 1977). Aid has been introduced previously in such forms as learning objectives (Rothkopf \& Kaplan, 1972), text cuing (Lorch \& Chen, 1986), and, especially, feedback (Barringer \& Gholson, 1979). While the outcomes of these studies have not been systematically positive and uniform, the basic theme has remained unchanged. The reasons for this consistently axiomatic viewpoint regarding the efficacy of instructional evidence are varied and complex. In general, they seem to primarily reflect basic principles underlying the associationist view of learning, with its emphasis on outcomes rather than process analyses. Under these assumptions, assistance becomes part of a general systems approach to instructional management (Langer \& Keenan, 1984).

This study is one of a series of experiments begun in 1984 to explore the effects of feedback on text processing. Feedback is a common form of assistance, defined as some type of help provided subsequent to a response. Whether the effects of feedback can be viewed in terms of controlling consequences or providing information is still a moot point (Anderson \& Faust, 1973; Barringer \& Gholson, 1979; Getsie, Langer, \& Glass, 1985). Our project was originally conceived as a search for parameters in instructional assistance rather than discourse processing per se. The outcomes have been less than uniform, however, requiring us now to consider, in a general

This paper is based in part on a conference report presented at the 1991 meeting of the Psychonomic Society in New Orleans, LA. Requests for reprints should be sent to Philip Langer, Institute of Cognitive Science, University of Colorado, Box 345, Boulder, CO 80309. way, the mechanisms of text processing (Langer, Keenan, $\&$ Culler, 1987). Our data would suggest that there are indeed limitations regarding impact of instructional assistance, especially in terms of the processes underlying the synthesizing of meaning from text.

In addition to the conclusions we have drawn from our project data, other researchers have argued that assistance might not necessarily have positive effects. Battig (1966) insisted that learning that was initially facilitated might not be the most desirable instructional outcome. Gallagher (1981) showed that feedback could hinder subsequent learning, while Sternberg and Ketron (1982) argued that student-generated strategies might prove superior to instruction-derived strategies. Indeed, Kulhavy (1977) stated that the more complicated feedback programs may themselves become learning tasks.

Our research program has utilized a paradigm based on the reconstruction of scrambled text. Assistance has been provided in the form of feedback, which either confirmed or disconfirmed the appropriateness of the sentence order developed during the reconstruction task. The passages were selected from regular texts and were not especially constructed for the experiment (Bransford \& Franks, 1971). Comprehension was assessed by comparing the order of the reconstructed text with the original sentence sequence and by using the more common retrieval measures of idea recall and sentence recognition. Our choice of this paradigm was based on the premise that the reconstruction of scrambled text is a process and therefore supposedly sensitive to the influence of instructional management. While scrambled text has been used extensively in the past as a variable, comparatively little has been done recently (Langer \& Keenan, 1984).

As noted, the interpretation of data based on the reconstruction paradigm has led us to consider more and more 
the nature of text processing itself. Interestingly, our more recent conclusions fit in with a model developed by Kintsch (Kintsch, 1974; Kintsch, 1988; van Dijk \& Kintsch, 1983). Kintsch (1988) argued that the initial text propositions are formed directly from the text itself but are then elaborated and integrated into a coherent text base representation, guided by the prior knowledge available. Comprehension is an iterative construction-integration process, and, obviously, both knowledge and text base change as a result of the processing. The model suggests that several layers of knowledge interact simultaneously with the task of understanding discourse. Evidence for the model may show a surface representation of verbatim text, a propositional representation of semantic meanings, and a situation representation of pragmatic interpretations. Kintsch, Welch, Schmalhofer, and Zimny (1990) went on to elaborate the model in terms of sentence memory. Linguistic elements and syntactic chunks (surface representation features) interact with propositions (semantic representations) and situation elements. These interact concomitantly and do not represent distinct phases as the phase term is commonly used (Shuell, 1991).

The situation representation was tested in a series of experiments by Perrig and Kintsch (1985). In that investigation, there were two descriptions of a mythical town called Baldwin. One consisted of a sequential set of instructions for driving through the town; the other was a spatial or geographic layout. Surface representation was detected by recognition of verbatim sentences; text-based representation was detected by propositional recall, and situation representation was assessed by inferences.

This argument for distinct levels of knowledge leads to questions about thought processes in reading that are of vital concern to educational researchers and practitioners. In terms of our own feedback research, it is important that we know what kinds of intervention are most facilitative for the discrete kinds of memorial representation postulated in this model. In turn, these are dictated by the instructional outcome specifically sought. In this study, the second in a series, we attempt to determine whether feedback type contributes differentially to subsequent retrieval demands, using two passages that describe the same situation but differ in textual form.

In the first study, we used a slightly modified version of the Perrig and Kintsch description of their mythical town (Langer, Keenan, \& Nelson, 1991). To accommodate the changes, the town was called Mapleton. Each version was 24 sentences long. Again, one was a spatial or survey description, and the other was a route version that guided the driver through town. Both descriptions were presented in scrambled form to three groups of subjects. One group of subjects reconstructed the scrambled passages, with assistance provided in the form of limited confirmation requests as to the correct order of sentence placement. A second group reconstructed the scrambled text without feedback. A third group simply read the scrambled pas- sages twice. We assessed comprehension by comparing the order of the reconstructed text sentences with the original and by using the measure of recall and recognition.

Recall scores, defined as the proportion of atomic propositions correctly remembered, were statistically greater for the route version across conditions, paralleling the Perrig and Kintsch (1985) findings. Recognition scores, defined as the correct identification of old sentences from paraphrases, were not different across conditions. It may simply be that college students are subjects who, when called upon to read an unknown text, have developed to an art form memorial strategies to generate a valid surface representation. There was no feedback $\times$ text interaction, which we had assumed might be present. Because of the absence of any interaction, we decided to strengthen the feedback provided in terms of appropriateness by using a map. This permitted us to compare one type of feedback (map) with another (token).

\section{Method}

In this second project, we used the same two descriptions of Mapleton. Again, the route version was a set of instructions to guide a driver through the town. The survey version gave a spatial (i.e., geographic) description of the layout of the town. Each version was presented in a 24-sentence passage. The actual canonical order in which features were described in both versions was determined by the route version.

The route version contained 497 words, forming 226 atomic propositions. The survey version had 503 words and 227 propositions. The sentences for each version were typed singly on $3 \times 5$ in. cards, with two random orders for each version. The subjects were asked to rearrange the cards so the reconstructed order made sense to them. A slotted board was provided to aid the subject in sorting the cards. The subjects were general psychology students who participated as part of the course requirements. They were randomly assigned to the four treatment cells created by the two passages (route or survey) and two forms of assistance (token or map).

In the reconstruct-token confirmation condition, the subjects were given 25 tokens. While arranging the cards, the subject could use one token, up to a total of 25 requests, to confirm the placement of a card. The appropriateness of a placement was determined by the sentence immediately preceding it. To be given positive confirmation, the order of placement had to be such that, in the original order, the sentence in question did indeed follow the one preceding it on the board. Even if the original text in the sentences was actually separated by several other sentences, the subject was told that the placement was correct.

In the reconstruct-map assistance condition, the subjects who reconstructed the text sequence had access to a map that could be used to modify their sentence order. They were given five tokens and could use a token to look at the map for up to $10 \mathrm{sec}$. They were given access to a map after they had placed the sentence in question; they could modify this sentence placement after viewing the map. In both conditions, the subjects would continue to reconstruct the passages even after the request tokens were used up.

Following the reconstruction processing, the subjects were first asked to recall as much of the passage as they could remember without regard to order. They then took a recognition test consisting of sentences from a list in which half the sentences were paraphrased. The order of the reconstructed passage was recorded while the subjects were taking the recall and recognition measures.

\section{Results}

As in the previous research, the major measures of comprehension were recall, recognition, and concordance of 
sentence order with the original passages. Recall was defined as the number of propositions retained, recognition was defined as $d^{\prime}$, and concordance was reported as $\tau$.

For recall, there were main effects for both version and feedback. The mean number of propositions recalled for the route version $(44.08)$ was significantly greater than for the survey $(30.14)[F(1,95)=15.05, p<.001]$. This paralleled the Perrig and Kintsch (1985) findings, even given the significantly different task of reconstructing scrambled text. The mean for map feedback (41.22) was significantly greater than for token assistance (33.22) $[F(1,95)=4.85, p<.03]$. There was no statistically significant interaction between version and feedback. However, there were some simple effects that were in the predicted direction. There was some facilitation for map assistance, relative to tokens, for the route version. The map-assisted survey proposition recall mean (31.54) was slightly higher than the token-assisted survey recall mean (28.80). In contrast, the map-assisted route recall mean (50.52) was proportionally greater than the token-assisted route mean (37.64).

Our $d^{\prime}$ recognition analysis showed no differences between groups. Unlike previous research that found differences, we have attributed this result to our use of an inadequate number of sentences.

Our third measure of comprehension was the effect of order of sentence agreement between the reconstructed passage sentence and the original $(\tau)$ on recall and recognition. Recall was significantly related to $\tau(r=.22$, $p<.05)$, but recognition $\left(d^{\prime}\right)$ was not $(r=-.12$, n.s. $)$. These findings are not dissimilar to what we have observed in the past. Interestingly, concordance was not significantly related to feedback $(r=-.18$, n.s. $)$, confirming our previous argument that the developing text structure, at least in terms of semantic representation, need not necessarily replicate the original in order to achieve a satisfactory level of recall and recognition.

\section{Conclusions}

Our previous research using scrambled discourse to establish the impact of instructional assistance has, not unexpectedly, drawn fire from reading researchers in education. The use of the reconstructed text paradigm in a laboratory setting has proved to be an anathema to some researchers focused on intact classroom reading situations. However, the use of the Perrig and Kintsch (1985) passage, albeit modified, and the presence of similar findings, makes us breathe a bit easier. Clearly, text processing can be analyzed under controlled and limiting experimental procedures, as opposed to the many field-based shotgun approaches, where the absence of significant knowledge about the vast array of noncontrolled variables invalidates any reliable conclusions regarding cause and effect.

From an instructional viewpoint, we found that with the use of different forms of assistance, we were more clearly able to establish what we have argued for so long. That is, the value of any kind of feedback is related more closely to the specific text content and memorial task demands on the reader than previous researchers were willing to admit. Much of the prior work, especially in educational research, has indeed been rather simplistic in terms of any systematic analysis of the problems we are exploring (Langer \& Keenan, 1984). The data in this study would seem to lend support to our argument. It would appear, for example, that the information provided by the map assistance was much more amenable to manipulation of the developing route text version, relative to the sequentially driven token assistance. Our belief still remains that instructional assistance based on management principles rather than an analysis of the learning mechanisms involved, especially in text processing, is a major strategic error.

\section{REFERENCES}

ANDERSON, R. C., \& FAUST, G. W. (1973). Educational psychology: The science of instruction and learning. New York: Dodd Mead.

BARRINGer, C., \& GHolson, B. (1979). Effects and types of combinations of feedback upon conceptual learning by children: Implications for research in academic learning. Review of Educational Research, 49, 456-478.

BATTIG, W. F. (1966). Facilitation and interference. In E. A. Bilodeau (Ed.), Acquisition of skill. New York: Academic Press.

Berliner, D. C., \& Rosenshine, B. (1977). The acquisition of knowledge in the classroom. In R. C. Anderson, R. J. Spiro, \& W. E. Montague (Eds.), Schooling and the acquisition of knowledge (pp. 375404). Hillsdale, NJ: Erlbaum.

Bransford, J. D., \& Franks, J. J. (1971). The abstraction of linguistic ideas. Cognitive Psychology, 2, 331-350.

Gallagher, J. P. (1981). The effectiveness of man-machine tutorial dialogue for teaching attribute blocks problem-solving skills with an artificial intelligence CAI system. Instructional Science, 10, 291-332.

Getsie, R., Langer, P., \& Glass, G. V. (1985). Meta-analyses of the effects of type and combination of feedback on children's discrimination learning. Review of Educational Research, 55, 9-22.

KINTSCH, W. (1974). The representation of meaning in memory. Hillsdale, NJ: Erlbaum.

KINTSCH, W. (1988). The role of knowledge in discourse comprehension: A construction-integration model. Psychological Review, 95, 163-182.

Kintsch, W., WelCh, D., SChmalhofer, F., \& Zimny, S. (1990). Sentence memory: A theoretical analysis. Journal of Memory \& Language, 29, 133-159.

Kulhavy, R. W. (1977). Feedback in written instruction. Review of Educational Research, 47, 211-232.

LANGer, P., \& KeEnan, V. (1984). Modification of current feedback strategies: A text synthesis approach (Tech. Rep. No. 127). Boulder: University of Colorado, Institute of Cognitive Science.

Langer, P., Keenan, V., \& Culler, J. (1987). Effect of content on feedback in processing discourse. Psychological Reports, 60, 1191-1200.

Langer, P., Keenan, V., \& Nelson, S. (1991). Sentence-order feedback during processing of sequential or spatial texts. Bulletin of the Psychonomic Society, 29, 31-32.

LORCH, R. F., JR., \& CHEN, A. H. (1986). Effects of number signals on reading and recall. Journal of Educational Psychology, 78, 263-270.

Perrig, W., \& KINTsCh, W. (1985). Propositional and situational representation of text. Journal of Memory \& Language, 24, 503-518.

RothKOPF, M. C., \& KAPLAN, R. (1972). Exploration of the effects of density and specificity of instructional objectives on learning from text. Journal of Educational Psychology, 63, 293-302.

Shuell, T. S. (1991). Phases of meaningful learning. Review of Educational Research, 60, 531-547.

Sternberg, R. N., Ketron, D. L. R. (1982). Selection and implementation of strategies in reasoning by analogy. Journal of Educational Psychology, 74, 399-441.

van DuK, T. A., \& KINTsCh, W. (1983). Strategies of discourse processing. New York: Academic Press.

(Manuscript received February 15, 1991.) 